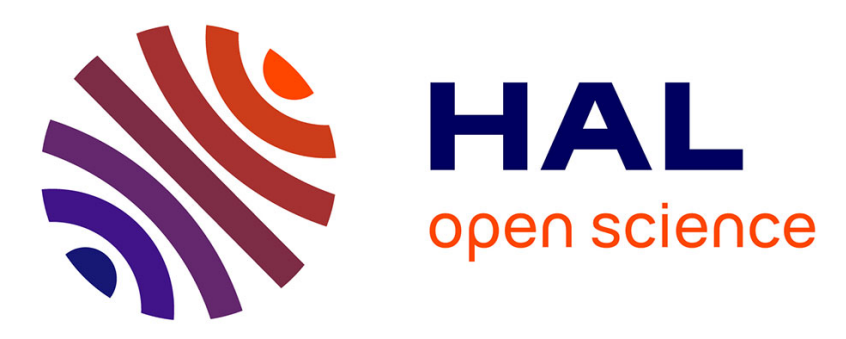

\title{
Bayesian optimization-based algorithm to improve the quality of transmission estimation
}

\author{
Reda Ayassi, Ahmed Triki, Maxime Laye, Esther Le Rouzic, Noel Crespi,
} Roberto Minerva

\section{> To cite this version:}

Reda Ayassi, Ahmed Triki, Maxime Laye, Esther Le Rouzic, Noel Crespi, et al.. Bayesian optimizationbased algorithm to improve the quality of transmission estimation. Advanced Photonics Conference 2021, Jul 2021, Washington DC (virtual), United States. pp.NeF2B.3, 10.1364/NETWORKS.2021.NeF2B.3 . hal-03254064

\section{HAL Id: hal-03254064 https://hal.science/hal-03254064}

Submitted on 8 Jun 2021

HAL is a multi-disciplinary open access archive for the deposit and dissemination of scientific research documents, whether they are published or not. The documents may come from teaching and research institutions in France or abroad, or from public or private research centers.
L'archive ouverte pluridisciplinaire HAL, est destinée au dépôt et à la diffusion de documents scientifiques de niveau recherche, publiés ou non, émanant des établissements d'enseignement et de recherche français ou étrangers, des laboratoires publics ou privés. 


\title{
Bayesian Optimization-Based Algorithm to Improve the Quality of Transmission Estimation
}

\author{
Reda Ayassi ${ }^{1,2}$, Ahmed Triki ${ }^{1}$, Maxime Laye ${ }^{1}$, Esther Le Rouzic $^{1}$, Noel Crespi ${ }^{2}$, Roberto Minerva ${ }^{2}$ \\ 1. Orange Labs, 2 Avenue Pierre Marzin, 22300 Lannion, France \\ 2. Institut Polytechnique de Paris, Institut Mines-Telecom, Telecom SudParis, France \\ reda.ayassi@orange.com
}

\begin{abstract}
We propose a Bayesian Optimization-based algorithm to assess the optical parameters that are taken as input by the QoT computation tool. The method reduces the error in computed OSNR down to $0.07 \mathrm{~dB}$. () 2021 The Author(s)
\end{abstract}

\section{Introduction}

The high-order modulation aims to push the optical transport technology to their limits. In such a system where every tenth of a dB is precious, an accurate calculation of the Quality of Transmission (QoT) becomes essential more than ever. The analytical tools for QoT estimation are based on models that assume idealized systems and are not general enough to model the high diversity of the optical transport networks [1]. Moreover, the assessment of optical parameters used as input of those analytical tools is based on values provided by the equipment vendor and does not take into account the actual field factors (e.g., aging). To cope with the inaccuracy of the QoT estimation, design margins are added by operators which lead to under-utilization of the available capacities. Recent studies have focused on Machine Learning (ML) to improve the accuracy of QoT estimation [2]. While the subject of some ML-based solutions has been to replace the existing analytical tools [3], others have focused on improving the accuracy of the analytical computation of the QoT. This could be done either by assessing nonlinear impairments that are complex to model analytically [4] or by reducing the error in the input parameters [5]. In this paper, we propose a Bayesian optimization-based algorithm to find a set of input parameters values that improve the accuracy of QoT estimation tools. The novelty in this work is that our approach is independent of the QoT estimation tool and can be applied to any uncertain parameter. Therefore, we formulate our approach as an optimization problem and we assume that its objective function is black box. Without a closed form expression of the QoT computation function, the gradientbased algorithms cannot be used as in [5], so we have to use a search-based algorithm instead.

\section{Problem formulation and simulation set up}

We consider $G=(N, L)$ as a graph that abstract the optical network where vertices $\mathrm{N}$ present the set of optical nodes and edges L present the set of links. We assume each node is composed of reconfigurable optical add and drop multiplexer (ROADM) and multiple transponders. Each link consists of several fiber spans. We denote the set of lightpaths that are deployed on the network as $P=\left\{p_{i} \mid i \in[0, n]\right\}$. We denote the real value (i.e., measured) of a QoT indicator for lightpath $p_{i}$ by $Q_{i}$ and its estimated value using the analytical model by $\widetilde{Q}_{i}$. In this study, we suppose that the difference between $\tilde{Q}_{i}$ and $Q_{i}$ is only due to the inaccuracy in the values of some parameters. Therefore, we split the input parameters into two sets, certain $\mathrm{C}$ and uncertain $X=\{j \mid j \in[0, l]\}$ where $l$ is the total number of uncertain parameter. We denote $x_{j}$ the real value of the parameter $j$, and $\tilde{x}_{j}$ its theoretical value. Reducing the difference between $x_{j}$ and $\tilde{x}_{j}$ leads to a more exact modeling of the network state, and consequently reduces the difference $\left|Q_{i}-\tilde{Q}_{i}\right|$ (i.e., more accurate QoT estimation). Accordingly, the problem of reducing the error in QoT estimation can be considered as a multi-objective general optimization problem with the goal to minimize the set of objective functions $\left\{f_{i}(C, X)=\right.$ $\left.\left|Q_{i}-\tilde{Q}_{i}\right| \mid i \in[0, n]\right\}$, where $n$ is the total number of lightpaths.

We consider three types of uncertain parameters: the amplifier Noise Figure (NF), the loss due to connectors (CL) and the loss due to fiber fusing (FL). In this paper, we focus on minimizing the OSNR error; nevertheless, the same model could be used to improve other QoT indicators. In order to reduce the complexity of the multi-objective optimization (i.e., one objective function per lightpath), we choose to aggregate all the objective functions into a single one using the root mean squared error (RMSE) operator. We use then the Bayesian optimization algorithm that is able to reduce the number of objective function evaluations needed to reach the optimum based on the cumulated knowledge during the iterations of the algorithm. To test this approach, we assume a five-node network topology as represented in Fig. 1 (c) and we use an analytical tool (the GNPy tool [6]) to both simulate the initial state of the network, as well as to compute the objective function after every iteration. We consider different lengths of fiber spans 
$(50,75,80$ and $100 \mathrm{~km})$. The amplifier gains are automatically set to compensate the fiber loss at the power optimum. Each fiber span is split into 4 fiber sections to take into account 3 FL parameters per span.

3. Learning process and results discussion
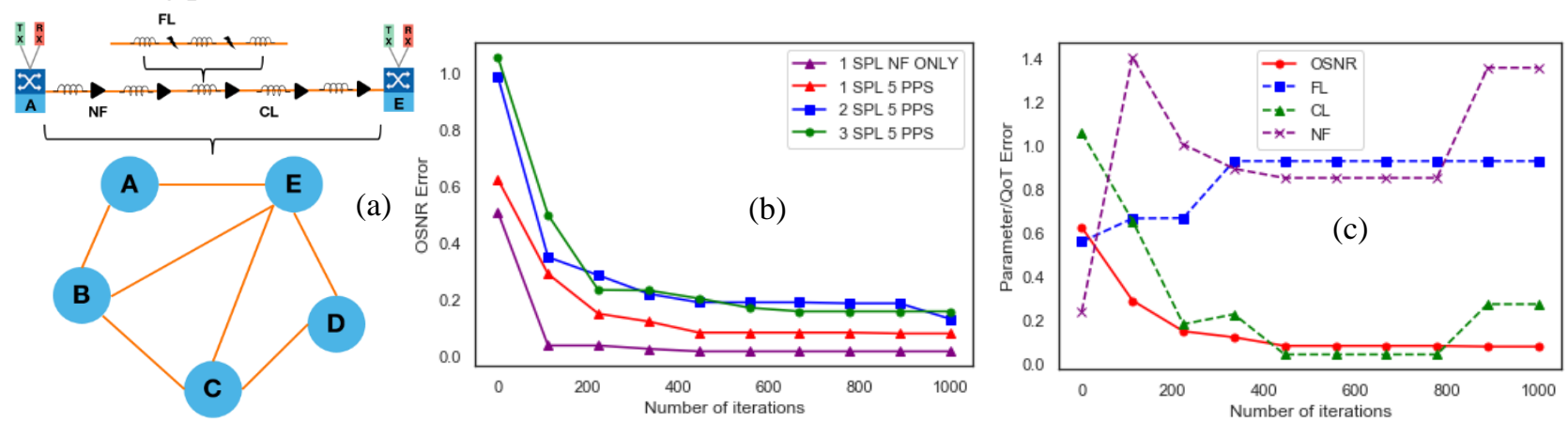

Fig.1. (a) 5-Node network topology used to test the learning process (b) Evolution of OSNR error throughout the optimization, (c) Evolution of OSNR and parameters errors in the case of 1 SPL and 5 PPS

In order to evaluate our Bayesian-based approach, the target values $x_{j}$ are first sampled according to a uniform distribution from fixed value intervals: for NF $x_{j} \in \Delta_{N F}$, for CL $x_{j} \in \Delta_{C L}$ and for FL $x_{j} \in \Delta_{F L}$. Then, the initial uncertain values $\tilde{x}_{j}$ are emulated by adding uniform noise around $x_{j}$ so that, for NF: $\tilde{x}_{j} \in\left[x_{j}-2, x_{j}+2\right] \cap \Delta_{N F}$, for $\mathrm{CL}: \tilde{x}_{j} \in\left[x_{j}-1.5, x_{j}+1.5\right] \cap \Delta_{C L}$ and for FL: $\tilde{x}_{j} \in\left[x_{j}-1, x_{j}+1\right] \cap \Delta_{F L}$. The algorithm takes as input $\tilde{x}_{j}$ and its search bounds (i.e., corresponding interval). After every iteration, the objective function $f(C, X)$ is evaluated using GNPy. Taking into account the results from all previous iterations, the algorithm generates then a new set of parameters $\left\{\tilde{x}_{j} \mid j \in[0, l]\right\}$ and tests it at the next iteration. We used 20 lightpaths for the learning process.

We applied the algorithm in different scenarios in order to test its scalability by changing the number of spans per link (SPL) and the number of uncertain parameters per span (PPS) in the network. Fig.1 (a) shows the evolution of the OSNR error during the optimization in each scenario. After 500 iterations, the OSNR error is below $0.2 \mathrm{~dB}$ in all cases. We represent in Fig.1 (c) the evolution of the error generated by the three parameters alongside the error in OSNR in the case of 1SPL and 5PPS (i.e., 70 parameters). After 500 iterations, the OSNR error becomes inferior to $0.07 \mathrm{~dB}$, however the inaccuracy in the parameters remains high and the parameter error is evolving in random patterns and does not converge toward the target values. This means that the algorithm finds another global optimum that is different from the target one. As we suppose that no intermediate performance measurement is possible within the link, the Bayesian optimization provides alternative settings of parameters that generate the same QoT as the actual setting. As a result, our approach can improve QoT estimation accuracy, by providing an alternative set of parameters, and can be applied with a minimal number of QoT measurements.

\section{Conclusion}

We proposed a Bayesian optimization learning process to improve the accuracy of QoT estimation by assessing the values of uncertain parameters of the optical network. Our method is QoT computation tool-agnostic and supports several uncertain parameters. In this paper, we considered a set of three uncertain parameters, namely, amplifier noise figures, fiber connector loss and fiber fused loss. Results showed that applying the algorithm to scenarios having 70 uncertain parameters, the error in OSNR was reduced from $1 \mathrm{~dB}$ to $0.07 \mathrm{~dB}$.

\section{References}

[1] M. Ranjbar Zefreh et al., "Accurate Closed-Form Real-Time EGN Model Formula Leveraging Machine-Learning Over 8500 Thoroughly Randomized Full C-Band Systems," J. Light. Technol., vol. 38, no. 18, pp. 4987-4999, Sep. 2020

[2] R. Ayassi et al., "An Overview on Machine Learning-Based Solutions to Improve Lightpath QoT Estimation,” in 2020 22nd International Conference on Transparent Optical Networks (ICTON), Bari, Italy, Jul. 2020, pp. 1-4

[3] T. Panayiotou et al., "Centralized and Distributed Machine Learning-Based QoT Estimation for Sliceable Optical Networks," ArXiv190808338 Cs Eess, Sep. 2019

[4] Q. Zhuge et al., "Application of Machine Learning in Fiber Nonlinearity Modeling and Monitoring for Elastic Optical Networks," J. Light. Technol., vol. 37, no. 13, pp. 3055-3063, Jul. 2019, doi: 10.1109/JLT.2019.2910143.

[5] E. Seve et al., "Learning Process for Reducing Uncertainties on Network Parameters and Design Margins," J. Opt. Commun. Netw., vol. 10, no. 2, p. A298, Feb. 2018

[6] A. Ferrari et al., "GNPy: an Open Source Application for Physical Layer Aware Open Optical Networks," J. Opt. Commun. Netw., vol. 12, no. 6 , p. C31, Jun. 2020 